\title{
HIGH-RESOLUTION RADIO OBSERVATIONS OF A GIANT H II REGION IN CYGNUS
}

\author{
JACOB W. M. BAARS \\ Netherlands Foundation for Radio Astronomy, Radiosterrenwacht, Dwingeloo, The Netherlands \\ and \\ HEINRICH J. WENDKER \\ Hamburger Sternwarte, Hamburg-Bergedorf, F.R.G.
}

\begin{abstract}
Westerbork observations of several large $\mathrm{HII}$ regions in the Cygnus-X region are described. IC 1318 appears to be a giant $\mathrm{H}$ II region, and two nearby sources may be genetically related to it.
\end{abstract}

\section{Introduction}

The highest angular resolution for observations of $\mathrm{H}$ II regions with a single antenna was obtained a few years ago by Schraml and Mezger (1969). They used the NRAO 43-m telescope at 2-cm wavelength, which resulted in a half-power-width of the main beam of about 2'. The results, particularly those on the nebula W49 (Mezger et al., 1967), indicated that, given a sufficiently narrow antenna beam, many interesting details would be seen in $\mathrm{H}$ II regions of small overall angular size.

Consequently such observations have been undertaken with interferometers, e.g., the Cambridge 'One-Mile-Telescope'. (Wynn-Williams, 1971) and the NRAO threeelement interferometer (Webster et al., 1971). These two aperture-synthesis instruments are either extremely slow or incapable of covering the aperture plane completely. Therefore the observations have been restricted mainly to bright regions of small angular size (in most cases significantly smaller than the beam of the antenna pattern of the interferometer elements).

These observations have shown the existence of compact condensations ('bright knots'). They are thought to be excited from a star within and are considered as very young $\mathrm{H}$ II regions. In almost all cases the total flux density in the interferometer map is less than the total flux density measured with a single antenna. This indicates that the source contains angular structure on a scale large enough to be resolved by the shortest interferometer spacing.

The Synthesis Radio Telescope (SRT) at Westerbork, The Netherlands, delivers a completely sampled aperture plane from $36 \mathrm{~m}$ to $1458 \mathrm{~m}$ with $18-\mathrm{m}$ intervals in 4 half-days of observing time. This enables the study of sources of relatively large angular size with a very good resolution.

We therefore used this instrument for observations of several large $\mathrm{H}$ il regions in the Cygnus-X region. One aim was to study the hierarchy of sub-structures in these presumably old sources. The choice of the general region of the sky was influenced by the availability of plates in the $\mathrm{H} \alpha$ line, enabling the study of the relation between radio and optical structures and the absorption across the nebulae. 


\section{The Radio Observations}

Figure 1 shows part of the Cygnus-X region as observed by Wendker (1970) at $11-\mathrm{cm}$ wavelength with an angular resolution of $11^{\prime}$. The SRT observations have been done in the region of IC $1318 \mathrm{~b}$ and $\mathrm{c}$, the companion sources north $(\mathrm{G} 79.3+1.3)$ and south $(G 78.1+0.6)$ of these, the so-called $\gamma$-Cygni source, and the area around G78.2-0.4 and G78.0+0.0. Note that the northern and southern companions are much smaller and appear more symmetrical than the regions IC $1318 \mathrm{~b}$ and c. Figure 2 is a copy of the $\mathrm{H} \alpha$ plate of the region; IC $1318 \mathrm{~b}$ and $\mathrm{c}$ are separated by a large lane of dust, while the northern and southern sources are highly obscured. We adopt a distance of $1.5 \mathrm{kpc}$ to all the sources (Dickel et al., 1969).

We turn now to the Westerbork observations of IC $1318 \mathrm{~b}$, at $21 \mathrm{~cm}$ with a synthesized beam of $30^{\prime \prime} \times 45^{\prime \prime}$. Figure 3 shows the resulting contour map. Integration of the map indicates that only $15 \%$ of the flux density measured with a single antenna is present. The map appears to be a blend of about 10 substructures of the size of a few

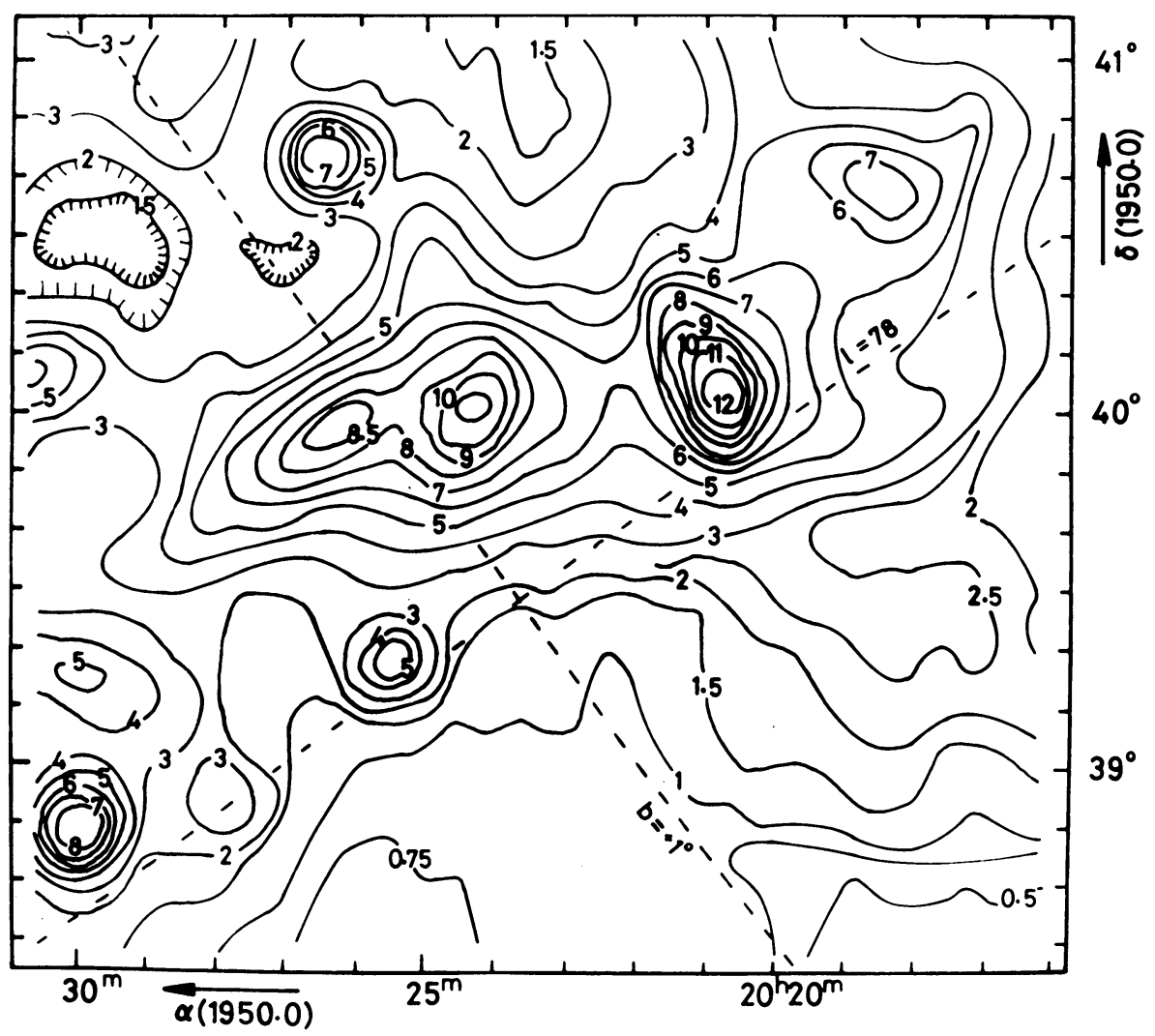

Fig. 1. Part of $11-\mathrm{cm}$ map of Cygnus-X region obtained by Wendker at $\lambda=11 \mathrm{~cm}$ with a resolution of $11^{\prime}$. The brightest source is the $\gamma$-Cygni source; to the east of it are IC $1318 \mathrm{~b}$ and $\mathrm{c}$ with their northern and southern companions. 


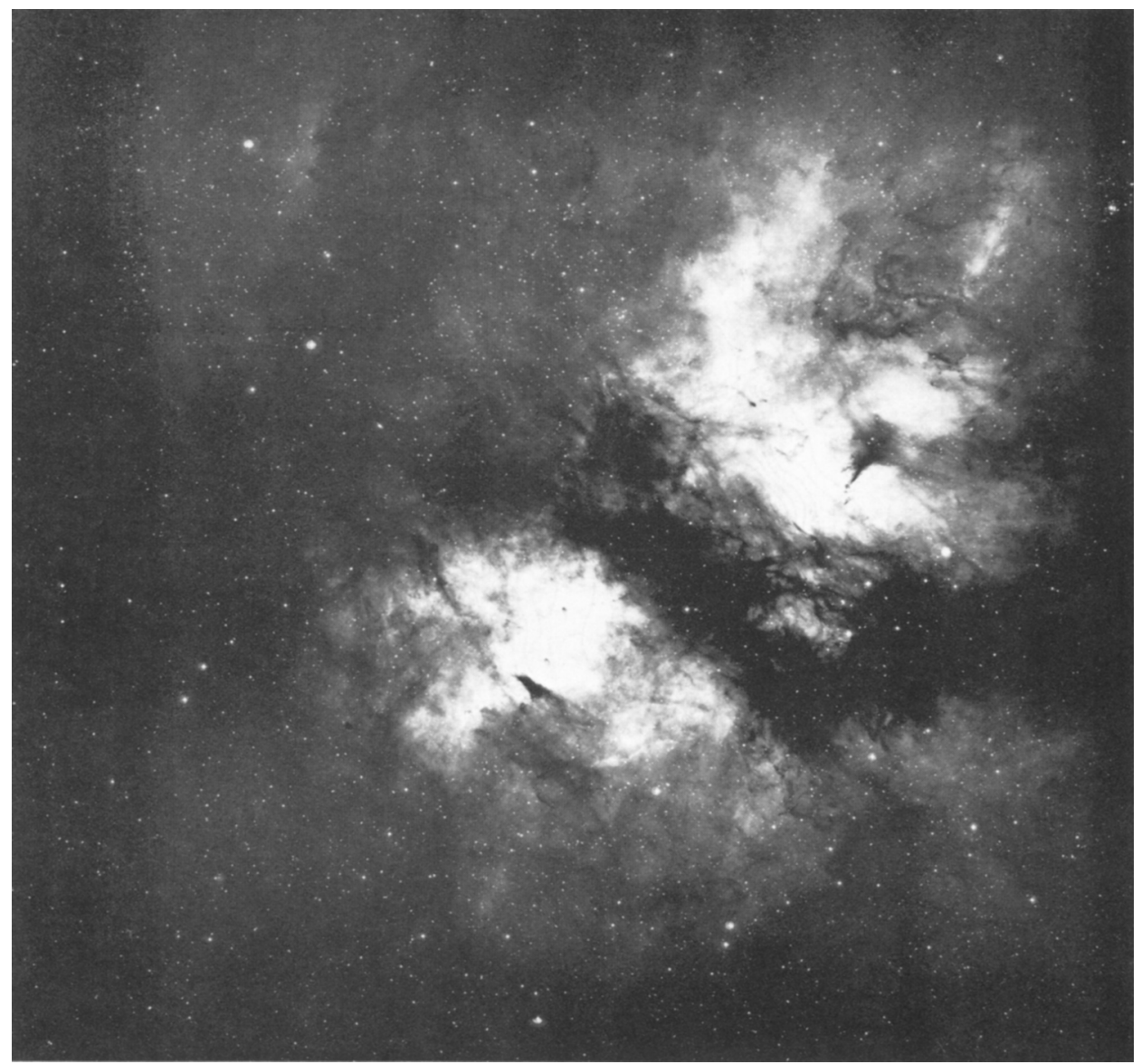

Fig. 2. A photograph in $\mathrm{H} \alpha$ of IC $1318 \mathrm{~b}$ and c. Note the conspicuous dust lane crossing the region. Photograph by H. R. Dickel and H. J. Wendker. Courtesy of Hale Observatories.

arcmin. The half-power angular width of the source, as deduced from the singleantenna observations is $20^{\prime} \times 30^{\prime}$, beyond the region where the SRT sees significant radiation. An obvious conclusion is that the greater part of the source's flux density comes from a large, smooth component which is at best only partially seen by the smallest interferometer spacing of $36 \mathrm{~m}$.

To check this quantitatively we have used the source parameters from the $11-\mathrm{cm}$ map as starting parameters. Since the sources are known to be optically thin at 21 and $11 \mathrm{~cm}$ no serious error can arise in doing this. By an iterative least-squares fit to the complex visibilities we found the parameters of those sources which dominate the 36- $\mathrm{m}$ and 54-m spacings of the SRT observations. In comparing the final fit with the single dish data we find that the SRT sources fit only the upper part of the single antenna scans. This suggests that a component exists with size $>1^{\circ}$ which is thus completely absent in the $36-\mathrm{m}$ spacing but contributes heavily to the single-antenna 


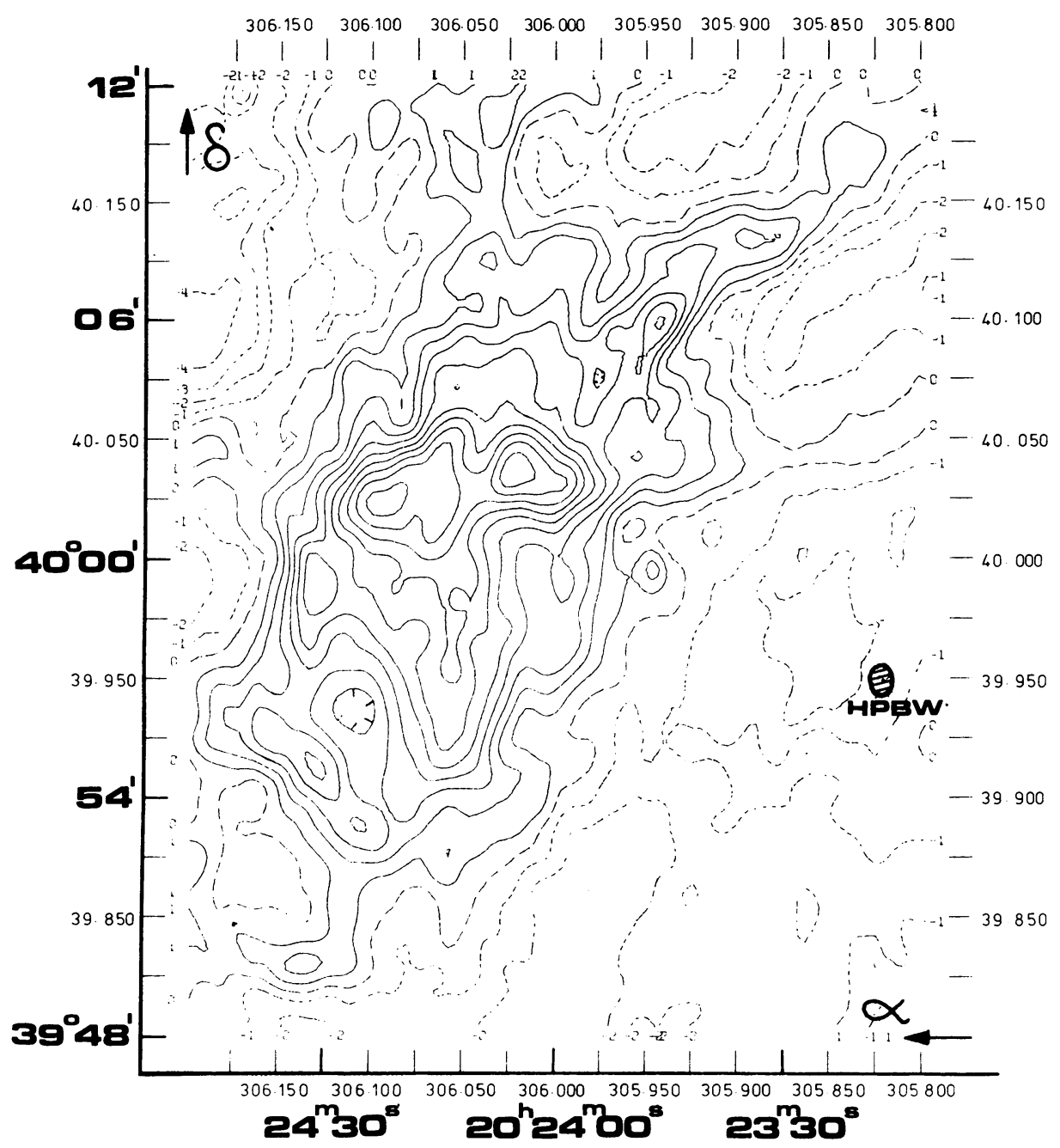

Fig. 3. IC $1318 \mathrm{~b}$ as observed with the SRT at $\lambda=21 \mathrm{~cm}$ with a synthesized beam of $30^{\prime \prime} \times 45^{\prime \prime}$. The full contours are 1(1) $10 \mathrm{~K}$ in brightness temperature, the dashed contours are negative responses. The observed size of the source is much too small, because of the loss of information on the large-scale structure in the interferometer output.

flux density. Introducing such a component in the single-dish data together with the components found from the two smallest spacings of the interferometer we obtained a reasonable fit to the single-dish data. We find a half-width of $75^{\prime}$ and a flux density of about $300 \mathrm{Jy}$ for this large extended component. Almost all of this component was considered to belong to the unresolved background contained in the $11-\mathrm{cm}$ data by Wendker (1970).

We conclude that we see the following hierarchy of angular structures in IC $1318 \mathrm{~b}$ :

(i) an extended envelope of size $75^{\prime}$; 
(ii) the intermediate sources, dominating the 11-cm data, of size $15^{\prime}-30^{\prime}$;

(iii) the finer scale substructure, seen only with the SRT, of size less than $5^{\prime}$.

The ratios of flux densities are about: (i):(ii):(iii) $=10: 3: 10^{-2}$.

Quite a different picture emerges in the case of the source $\mathrm{G} 79.3+1.3$, the northern companion of IC 1318b. SRT maps at $6 \mathrm{~cm}$ and $21 \mathrm{~cm}$ are available for this source. Figure 4 shows the 6-cm map. Here small-scale components stand out clearly. They

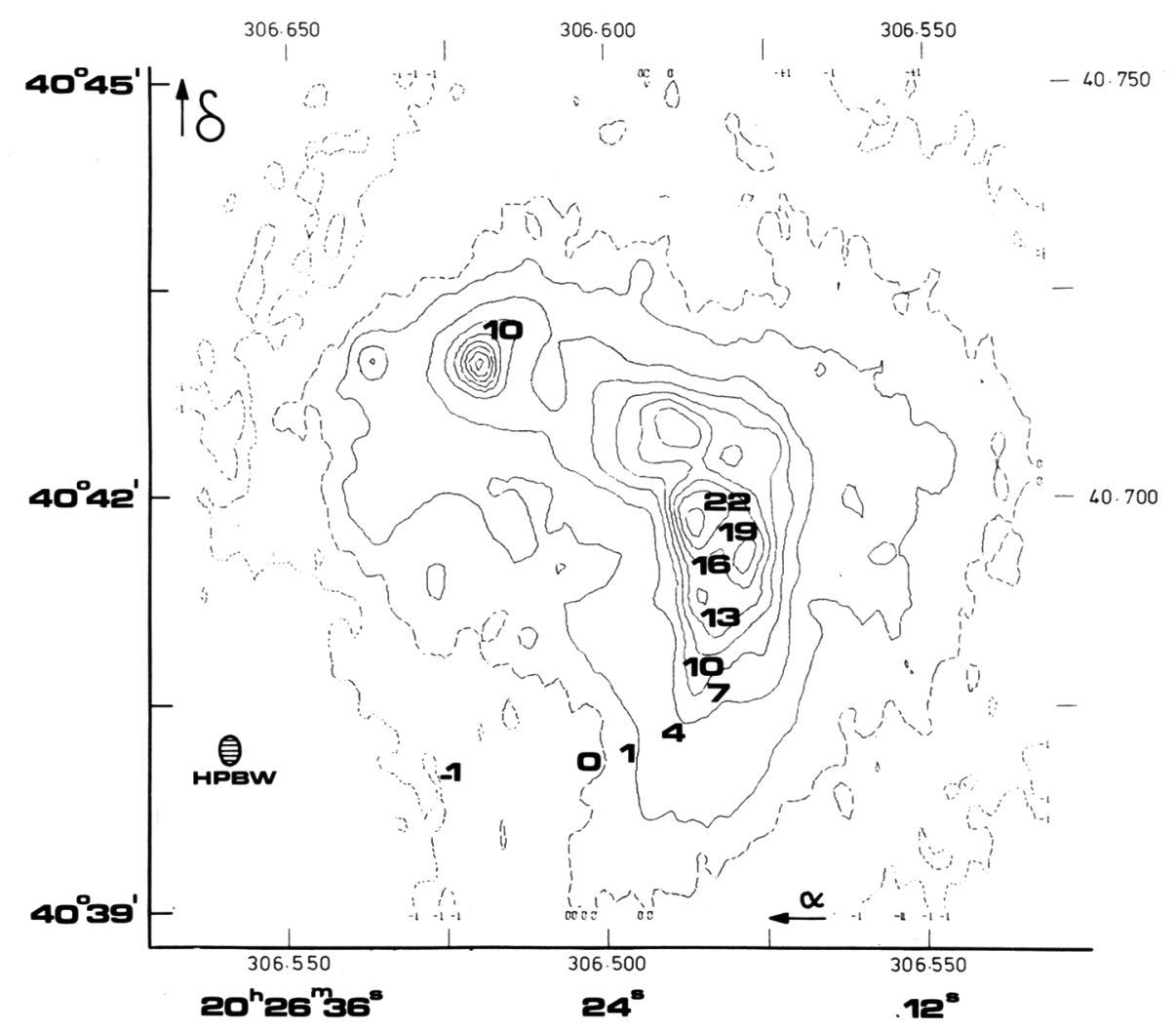

Figs. $4 \mathrm{a}-\mathrm{b}$. Contour map of $\mathrm{G} 79.3+1.3$, observed at $\lambda=6 \mathrm{~cm}$ with a resolution of $6^{\prime \prime} \times 10^{\prime \prime}$. Contour values are approximately in kelvins in equivalent brightness temperature. There are at least seven smallscale components.

are rather symmetrical in appearance and of small angular size. A comparison with the single-antenna flux density shows that by far the largest percentage of the flux density is contained in the condensations. This is very different from IC 1318. However, an analysis similar to that described above shows that at $21 \mathrm{~cm}$ there is a weak extended component which is smooth and covers the total source area. This component is lost at $6 \mathrm{~cm}$, but the $6-\mathrm{cm}$ data do show a smooth component underlying the condensations which is of intermediate size. 


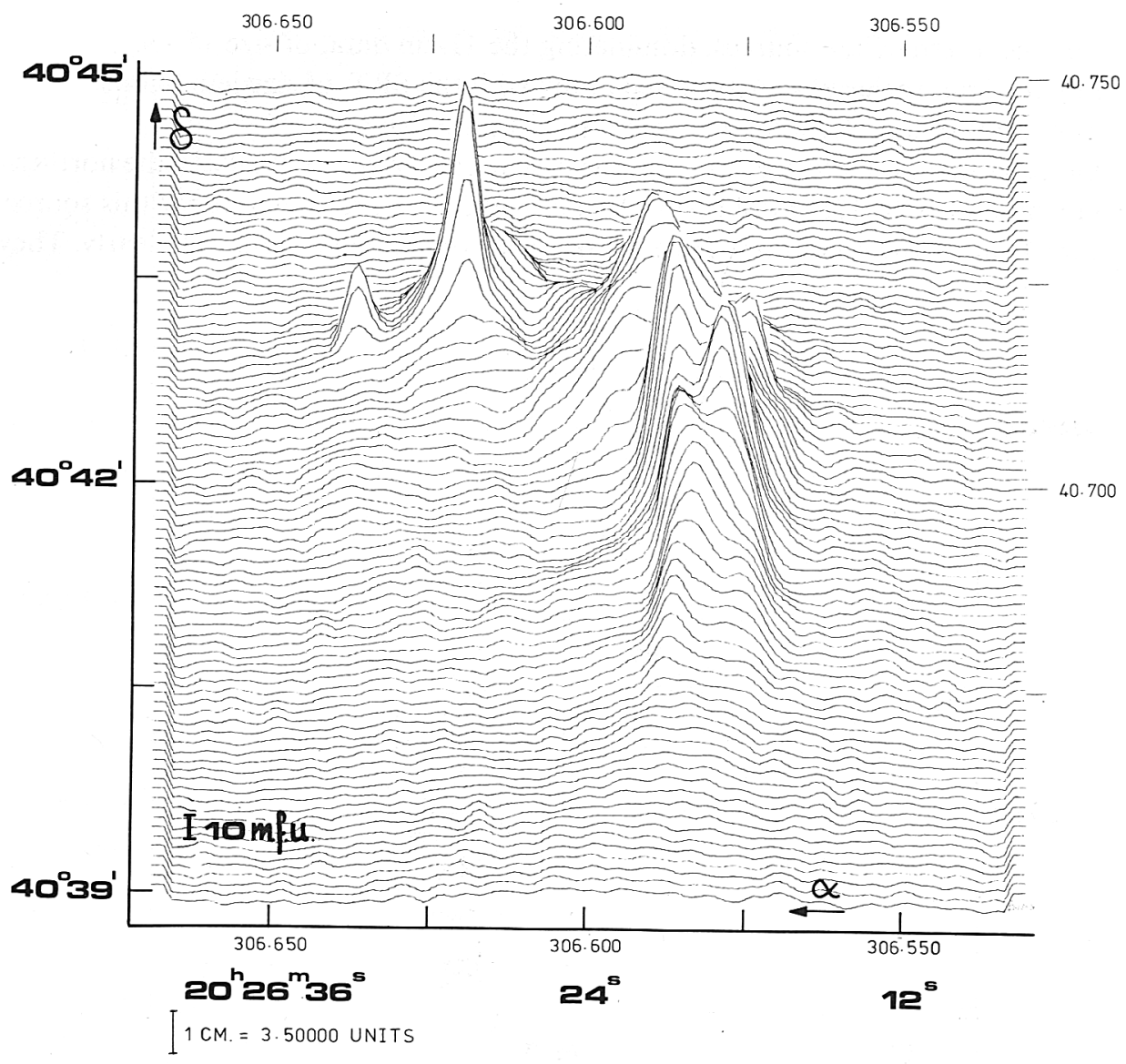

Fig. 4b. The same observation represented in a profile map.

\section{Discussion}

Results similar to IC $1318 \mathrm{~b}$ have been obtained for IC $1318 \mathrm{c}$ and in a preliminary form also for G78.2-0.4. In the same sense the southern companion and G78.0+0.0 resemble the northern companion of IC $1318 \mathrm{~b}$. Therefore the appearance of the two sources discussed above is not unique.

A main point from the above analysis is that the extended component of size $75^{\prime}$ covers both sources IC $1318 \mathrm{~b}$ and $\mathrm{c}$. This leads us to suggest that both nebulae IC $1318 \mathrm{~b}$ and $\mathrm{c}$ are in fact one $\mathrm{HII}$ region. This source then belongs to the class of giant $\mathrm{H}_{\mathrm{HI}}$ regions, as defined by Mezger (1970). If this suggestion is accepted it is probably of interest to point out that IC 1318 is then the giant $\mathrm{H}_{\text {II }}$ region closest to the Sun. Although it is intrinsically weaker than W49, the prototype of a giant Hur region, it contains all the hierarchy of substructures found in W49.

Physical parameters for the components, derived under the usual assumptions for $\mathrm{H}$ II regions, are listed in Table I. 
TABLE I

Physical parameters of IC $1318 \mathrm{~b}$ and c components

\begin{tabular}{lcccc}
\hline Parameter & Envelope & $\begin{array}{l}\text { Intermediate } \\
\text { component }\end{array}$ & $\begin{array}{l}\text { Small } \\
\text { component }\end{array}$ & $\begin{array}{l}\text { Total } \\
\text { source }\end{array}$ \\
\hline$T_{\mathrm{b}}(\mathrm{K})$ at $11 \mathrm{~cm}$ & 4 & 8 & 1 & 12 \\
Size $(\operatorname{arcmin})$ & 75 & 25 & 3 & 75 \\
Size in pc & 32 & 11 & 1.3 & 32 \\
$E\left(\mathrm{pc} \mathrm{cm}^{-6}\right)$ & 8400 & 17000 & 2100 & 25200 \\
$n_{\mathrm{e}}\left(\mathrm{cm}^{-3}\right)$ & 16 & 40 & 40 & 28 \\
$U\left(\mathrm{pc} \mathrm{cm}^{-2}\right)$ & 100 & 65 & 8 & 150 \\
Type of exciting star & O6/7 & O8 V & B0.5-1 V & O4 O5 \\
\hline
\end{tabular}

If the total source has one excitation center, a star of type O5 would be required for a smooth distribution of the gas. Since the observations clearly show this not to be the case, a somewhat hotter star, $\mathrm{O} 4$, is needed. There are no $\mathrm{O} 4$ stars observed as yet. The intermediate components in IC $1318 \mathrm{~b}$ and c can be excited by an $\mathrm{O} 8 \mathrm{~V}$ star alone. The small scale components are quite different from the compact $\mathrm{H}$ il regions studied, e.g., by Webster et al. (1971) or Habing et al. (1972). Their bright 'knots' require an O star as excitation center, while for our small components an early B star would suffice. In the present case however, we feel that the small-scale structure should be considered as an inherent part of the intermediate component, its appearance resulting from density gradients in the gas.

Now we discuss the sources of smaller angular size. As a prototype we take G79.3+ +1.3 , the northern companion of IC $1318 \mathrm{~b}$. The physical parameters of the components mentioned above are summarized in Table II.

TABLE II

Physical parameters of G79.3 +1.3 components

\begin{tabular}{lcccc}
\hline Parameter & Envelope & $\begin{array}{l}\text { Intermediate } \\
\text { component }\end{array}$ & $\begin{array}{l}\text { Condensation } \\
\text { (average) }\end{array}$ & $\begin{array}{l}\text { Total } \\
\text { source }\end{array}$ \\
\hline$T_{\mathrm{b}}(\mathrm{K})$ at $11 \mathrm{~cm}$ & 5 & 17 & 50 & 50 \\
Size $($ arcmin $)$ & 5 & 1.8 & 0.3 & 1.8 \\
Size in pc & 2.2 & 0.77 & 0.13 & 0.77 \\
$E\left(\mathrm{pc} \mathrm{cm}^{-6}\right)$ & 10300 & 35700 & 105000 & 105000 \\
$n_{\mathrm{e}}\left(\mathrm{cm}^{-3}\right)$ & 70 & 215 & 900 & 370 \\
$U\left(\mathrm{pc} \mathrm{cm}^{-2}\right)$ & 38 & 14 & 6 & 09 \\
Type of exciting star & O9 & B0.5 V & B1 V & O \\
\hline
\end{tabular}

It is seen that the condensations dominate the source G79.3+1.3. Certainly an O9 star could excite the total Hil region. However, it is hard to see how sharp small scale structure could develop in such a case.

We suggest on the basis of the morphology, as described in the previous section (see also Figure 4) that the condensations each have their own excitation center. This 
is contrary to our suggestion for IC $1318 \mathrm{~b}$ and c. A typical condensation here can be excited by a B1 V star. Thus this $\mathrm{H}$ II region could represent a very young association of B stars, so young that the stars have not yet lost their surrounding ionized shells. The situation is analogous to the young $\mathrm{O}$-star association thought to be responsible for the appearance of W49.

At this point it is tempting also to suggest that there is a genetic relationship between the giant $\mathrm{H}$ II region IC $1318 \mathrm{~b}$ and $\mathrm{c}$ and their northern and southern companions. In projection both companions are contained in the very extended envelope discussed in connection with IC $1318 \mathrm{~b}$ and $\mathrm{c}$. The whole complex would then be the first stage of the generation of an OB-star association, where the $\mathrm{H}$ II components of different size and density represent the formation of different members at different ages. A similar relationship is suggested by Israel et al. (1973) for a group of $\mathrm{H}$ II regions around $l=111^{\circ}$. A similar group may be represented by the $H$ II regions G81.6 +0.0 and $\mathrm{G} 81.7+0.5$ also situated in the Cygnus-X region (see Wendker, 1970).

\section{References}

Dickel, H. R., Wendker, H. J., and Bieritz, J. H.: 1969, Astron. Astrophys. 1, 270.

Habing, H. J., Israel, F. P., and de Jong, T.: 1972, Astron. Astrophys. 17, 329.

Israel, F. P., Habing, H. J., and de Jong, T.: 1973, Astron. Astrophys. 27, 143.

Mezger, P. G.: 1970, in W. Beckers and G. Contopoulos (eds.), 'The Spiral Structure of Our Galaxy', IAU Symp. 38, 107.

Mezger, P. G., Schraml, J., and Terzian, Y.: 1967, Astrophys. J. 150, 807.

Schraml, J. and Mezger, P. G.: 1969, Astrophys. J. 156, 169.

Webster, W. J., Altenhoff, W. J., and Wink, J. E.: 1971, Astron. J. 76, 677.

Wendker, H. J.: 1970, Astron. Astrophys. 4, 378.

Wynn-Williams, C. G.: 1971, Monthly Notices Roy. Astron. Soc. 151, 397.

Jacob W. M. Baars

Netherlands Foundation for Radio Astronomy,

Radiosterrenwacht

Dwingeloo, The Netherlands

Heinrich J. Wendker

Hamburger Sternwarte, Hamburg/Bergedorf, F.R.G.

\section{DISCUSSION}

Terzian: What distance did you adopt for the source? Does it correspond well with the kinematic distance from recombination lines?

Baars: We adopted $1.5 \mathrm{kpc}$; think it agrees. 